\title{
A priori grid quality estimation for high-order finite differencing
}

\author{
Ryu Fattah ${ }^{\mathrm{b}}$, David Angland ${ }^{\mathrm{a}, *}$, Xin Zhang ${ }^{\mathrm{b}}$ \\ ${ }^{a}$ Faculty of Engineering and the Environment, University of Southampton, Southampton, \\ $U K$. \\ ${ }^{b}$ Department of Mechanical and Aerospace Engineering, The Hong Kong University of \\ Science and Technology, Clear Water Bar, Kowloon, Hong Kong (SAR), China.
}

\begin{abstract}
Structured grids using the finite differencing method contain two sources of grid-induced truncation errors. The first is dependent on the solution field. The second is related only to the metrics of the grid transformation. The accuracy of the grid transformation metrics is affected by the inverse metrics, which are spatial derivatives of the grid in the generalised coordinates. The truncation errors contained in the inverse metrics are generated by the spatial schemes. Fourier analysis shows that the dispersion errors, by spatial schemes, have similarities to the transfer function of spatial filters. This similarity is exploited to define a grid quality metric that can be used to identify areas in the mesh that are likely to generate significant grid-induced errors. An inviscid vortex convection benchark case is used to quantify the correlation between the grid quality metric and the solution accuracy, for three common geometric features found in grids: abrupt changes in the grid metrics, skewness, and grid stretching. A strong correlation is obtained, provided that the grid transformation errors are the most significant sources of error.
\end{abstract}

Keywords: computational aeroacoustics, computational fluid dynamics, high-order, finite-differencing, truncation error, grid quality

\footnotetext{
*corresponding author

Email address: D. ANGLAND@soton.ac.uk (David Angland)
} 


\section{Introduction}

There is a requirement in computational fluid dynamics (CFD) and computational aeroacoustic (CAA) simulations for low dispersion and low dissipation numerical methods to accurately predict the generation, propagation, and interaction of acoustic, entropic and vortical disturbances. This requirement can be satisfied by using high-order finite differencing (FD) methods, which have been applied in various CFD and CAA studies [1, 2]. Finitedifferencing schemes are derived from a Taylor series and contain truncation errors. The amplitude of the truncation error may depend on the solution field and on the grid coordinates. The latter is the source of error that relates to the grid quality. Grids of higher quality generate smaller truncation errors, and contribute less to the total error, thereby providing a more accurate solution.

Abrupt changes in the grid spacing or grid line direction, for example along block interfaces, have been identified as sources of grid-induced errors $[3,4,5]$ and can lead to inaccuracies in the solution. Simple grid quality measures based only on local geometric properties have been suggested [6, 7]. However, in these examples a monotonic and strong correlation between the mesh quality metric and the solution accuracy was not obtained. The lack of a strong correlation may be caused by the presence of additional measures to ensure numerical stability. For example, Visbal and Giatonde [5] showed that low-pass filters, which can have similar effects on a numerical solution to artificial dissipation, can provide stable and accurate solutions in areas of the mesh where there are abrupt changes in the grid spacing or grid line direction. Solutions obtained under a strong influence of additional filtering or dissipation may reduce the impact of poor grid quality. However, stronger filters remove fine scale features from the solution field, thereby reducing the fidelity of the solution. Some filtering is inherently required for high-order methods to remove spurious modes. However, Colonius and Lele [8] emphasise that the removal of spurious waves should be attempted by improving the grid quality and enhancing the boundary condition accuracy rather than by using stronger filters or additional dissipation.

Many body-fitted curvilinear grids are generated in a two-step procedure. Firstly, the grid points along the block edges are mapped by a distribution function. The parameters of the distribution function may be manually specified, which may impact geometric properties such as the local grid spacing, and the grid stretching. In the second step the mesh enclosed by the block 
edges is generated. This may be obtained by transfinite interpolation (TFI), or by elliptic solvers. The latter method ensures a smooth grid, and therefore smooth grid transformation metrics [9]. Elliptic solvers may be coupled with additional control functions (which have manually tunable parameters) to achieve desirable grid clustering properties [9, 10]. The high level of manually specified parameters in the grid generation procedure, makes the grid quality prone to human errors. Therefore, an effective grid quality metric is required, in order to identify and resolve areas of poor grid quality efficiently. This is especially useful for manually generated structured, body fitted meshes typically used in high order finite difference CAA codes.

The quality of the mesh is often attributed to specific geometric properties such as the grid non-orthogonality $[11,12]$, or the aspect ratio $[7,13]$. For any structured grid, all geometric features can be expressed by the grid point distribution function. Present grid quality measures, that depend on this function, are based on the work by Vinokur [14], Mastin [3] and Thompson et al. [15]. In these methods, the effects of the grid geometry on the overall truncation error are evaluated by numerically approximating the leading order terms of the truncation error series. For example, Lee and Tsuei [11] applied this method to derive an equation to estimate the truncation errors of the convection terms in the two-dimensional Navier-Stokes equations. The equation requires the numerical evaluation of second and third derivatives in space by additional differencing schemes and is non-trivial to solve. Additionally, the equation can only be evaluated once a flow field solution is obtained. The formula does however account for the additional errors generated when applying different differencing schemes for the spatial derivatives and the grid metrics. Deng et al. [16] showed, for governing equations expressed in a strong conservation form, that this inconsistency of spatial schemes can violate the surface conservation law. This violation may generate artificial and undesirable source and sink terms in the governing equations that result in numerical instabilities, and degrade the robustness of high-order methods.

In the current work an alternate approach to truncation error analysis is outlined. The truncation errors for a generic spatial scheme are expressed by Fourier analysis, and are shown to hold similarities to the transfer function of spatial filters. This similarity is exploited to define a grid quality metric scalar that correlates to the truncation errors contained in the grid metrics. The proposed grid quality metric is applied on several grids containing three commonly found geometric features: abrupt changes in the grid metrics, 
grid skewness, and grid stretching. The results from these tests are used to determine the correlation between the grid quality metric and the solution error.

\section{Derivation of the grid quality metric}

The methodology to derive the grid quality metric is described for a 1$\mathrm{D}$ case. The application to higher dimensions is analogous. The 1-D wave equation in the generalised coordinate system is given by,

$$
\frac{\partial f}{\partial t}+U \frac{\partial f}{\partial \xi} \frac{\partial \xi}{\partial x}=0
$$

where $f$ is a scalar variable, $t$ is time, $U$ is the convection speed in the $x$ direction, and $\xi$ is the generalised coordinate. Equation 1 can be evaluated by spatial differencing schemes, and a temporal scheme, together with accurate boundary conditions. The term $\partial \xi / \partial x$ is the grid transformation metric that is evaluated by the inverse metric, which in turn is evaluated along the generalised coordinate. The first spatial derivative $(D)$ of scalar field $(f)$, with respect to $\xi$, can be evaluated by a generic spatial scheme expressed by,

$$
D_{i}+\sum_{Z=-N}^{M}\left(\alpha_{Z} D_{i+Z}\right)=\frac{1}{\Delta \xi} \sum_{z=-n}^{m}\left(a_{z} f_{i+z}\right),
$$

where the index $i$ is the point on the grid line, $-N$ and $M$ define the size of the implicit stencil, $-n$ and $m$ define the range of the explicit stencil, and the coefficients $\alpha$ and $a$ ensure the desired order of accuracy. Fourier analysis $[17,18]$ can be applied to the spatial scheme to obtain,

$$
j k^{*} \Delta \xi=\frac{\sum_{z=-n}^{m}\left(a_{z} \mathrm{e}^{j k \Delta \xi z}\right)}{1+\sum_{Z=-N}^{M}\left(\alpha_{Z} \mathrm{e}^{j k \Delta \xi Z}\right)},
$$

where $j=\sqrt{-1}$, and $k$ and $k^{*}$ are the original and modified wave numbers, respectively. This expression decomposes the truncation error of the spatial scheme into the contributions from discrete wave numbers. This approach to truncation error analysis does not require numerical evaluations of second and third derivative terms, that are required by other truncation error estimation methods [15]. The truncation error defined by Equation 3 is sensitive to the parameter $k \Delta \xi$, which is indicative of the grid resolution. The truncation error becomes significant as the parameter $k \Delta \xi$ exceeds a threshold wave 
number $k_{c}$, where the dispersion error exceeds $0.1 \%$ [19]. A similar error analysis applied to the temporal scheme [20] reveals that the truncation error will have an insignificant impact if the temporal resolution is sufficient.

In Equation 1, a stationary grid is considered, and the total numerical error is comprised of three different terms. The first term is a temporal derivative, which depends only on the solution field $f$. The temporal scheme will generate a truncation error that reduces with a smaller time-step. The remaining terms in Equation 1 are approximated by spatial differencing schemes. The first term, $\partial f / \partial \xi$, will generate large truncation errors if the solution field $f$ is resolved poorly along the curvilinear axis $\xi$. These errors can be reduced by performing a grid convergence study, where the grid refinement is focused on the areas containing large variations in $f$. This refinement requires prior knowledge of the solution field. Finally, the term $\partial \xi / \partial x$ is the metric of the grid transformation. This term will generate large truncation errors if the grid point distribution function $\xi$ is poorly resolved along the physical coordinate $x$. This source of error can also be reduced by mesh refinement. However, it can be done without prior knowledge of the solution field $f$. If the solution field is continuous and the time-step is sufficiently small, then the most significant source of error is likely due to the metrics of the grid transformation. Therefore, the focus of this work is to define a grid quality metric that correlates to the truncation errors associated with $\partial \xi / \partial x$.

\subsection{Truncation error estimation using filtering schemes}

The low-dissipation properties of high-order schemes may make the solution vulnerable to spurious modes. Spurious modes are typically of high wave number and may form due to poor boundary conditions, or due to dispersion errors. Spatial filters can be applied, which selectively damp high wave number modes in a solution field [17]. A generalised filtering scheme, derived from a truncated Taylor series, can be expressed by [17],

$$
\hat{\psi}_{i}+\sum_{Z=-M_{f}}^{N_{f}} \alpha_{z} \hat{\psi}_{i+Z}=\frac{1}{\Delta \xi} \sum_{z=-m_{f}}^{m_{f}} a_{z} \psi_{i+z},
$$

where $\psi$ and $\hat{\psi}$ are the unfiltered and filtered scalars, respectively. The order of the filter is determined by the coefficients $\alpha_{i}$ and $a_{i}$, and the size of the computational stencil is determined by the constants $-m_{f}, n_{f},-M_{f}$ and $N_{f}$. The filters are applied in the generalised coordinates where the grid spacing 
$(\Delta \xi)$ is uniform. By applying the Fourier analysis [17, 18], a filter transfer function is obtained,

$$
T(k)=\frac{\hat{\psi}(k)}{\psi(k)}=\frac{\sum_{Z=-m_{f}}^{n_{f}} a_{Z} \mathrm{e}^{j k Z}}{1+\sum_{z=-M_{f}}^{N_{f}} \alpha_{z} \mathrm{e}^{j k z}} .
$$

The transfer function defines the modulation to an input scalar field along discrete wave numbers. Low-pass filters have a cut-off wave number threshold, denoted by $k_{f}$, beyond which the filter entirely damps the mode amplitude. This threshold is determined by the order and the coefficients of the scheme. Therefore, an appropriate filtering scheme may be used to identify regions of a scalar field, which will likely generate significant truncation errors by the spatial differencing schemes. This method would be most effective, if the threshold values for $k_{c}$ and $k_{f}$ are similar. Additionally, the approximation of the truncation error by filtering schemes does not require any second or third derivatives to be evaluated.

\subsection{Grid quality metric}

The objective of the grid quality metric is to identify regions of the grid that are likely to generate significant grid-induced truncation errors. These sources of errors arise from the Jacobian $(J)$ and the standard metrics of the grid transformation, which are

$$
\begin{array}{ccc}
J^{-1}=\left|\frac{\partial(x, y, z)}{\partial(\xi, \eta, \zeta)}\right|, & \\
\xi_{x}=J\left(y_{\eta} z_{\zeta}-z_{\eta} y_{\zeta}\right), & \xi_{y}=J\left(z_{\eta} x_{\zeta}-x_{\eta} z_{\zeta}\right), & \xi_{z}=J\left(x_{\eta} y_{\zeta}-y_{\eta} x_{\zeta}\right), \\
\eta_{x}=J\left(y_{\zeta} z_{\xi}-z_{\zeta} y_{\xi}\right), & \eta_{y}=J\left(z_{\zeta} x_{\xi}-x_{\zeta} z_{\xi}\right), & \eta_{z}=J\left(x_{\zeta} y_{\xi}-y_{\zeta} x_{\xi}\right), \\
\zeta_{x}=J\left(y_{\xi} z_{\eta}-z_{\xi} y_{\eta}\right), & \zeta_{y}=J\left(z_{\xi} x_{\eta}-x_{\xi} z_{\eta}\right), & \zeta_{z}=J\left(x_{\xi} y_{\eta}-y_{\xi} x_{\eta}\right),
\end{array}
$$

and are expressed in terms of $\partial(x, y, z) / \partial(\xi, \eta, \zeta)$. Therefore, the truncation error contained within the grid metrics can be approximated by applying the spatial filtering schemes to the physical grid coordinates. A filtering operator is defined as,

$$
\hat{\Delta} \psi_{i}=\psi_{i}-\hat{\psi}_{i}
$$


which tends to zero as the scalar $\psi$ contains low wave number modes, and tends to $\hat{\Delta} \psi \rightarrow \psi$ as the scalar field contains only modes that are beyond the filter cut-off wave number $k_{f}$. In the present work sixth-order explicit [21] and sixth-order compact [22] filters are used to estimate the truncation errors generated by the fourth-order compact schemes [1,23]. The filtering operator for a scalar variable $\psi$, along a grid line of varying $\xi$, and at the index $i$ can be expressed as $\hat{\Delta} \psi_{i}$ in Equation 8. Similarly, the filtering operators for $\psi$ along grid lines of varying $\eta$ and $\zeta$ are expressed as $\hat{\Delta} \psi_{j}$ and $\hat{\Delta} \psi_{k}$, respectively. By applying the filter operators to the grid points along the generalised coordinates, the following nine terms are obtained

$$
\begin{array}{lll}
\hat{\Delta} x_{i}, & \hat{\Delta} x_{j}, & \hat{\Delta} x_{k}, \\
\hat{\Delta} y_{i}, & \hat{\Delta} y_{j}, & \hat{\Delta} y_{k}, \\
\hat{\Delta} z_{i}, & \hat{\Delta} z_{j}, & \hat{\Delta} z_{k} .
\end{array}
$$

The grid point distribution function for the $x$-coordinates along a grid line of varying $\xi$ is defined by $x_{i}$. The output from the filter operator, $\hat{\Delta} x_{i}$, will provide a measure of the truncation errors generated by the spatial scheme when evaluating $\partial x / \partial \xi$. Larger values in the terms $\hat{\Delta} x_{j}$ and $\hat{\Delta} x_{k}$ will similarly indicate greater truncation errors when the spatial scheme is applied along the $\eta$ and $\zeta$ directions, respectively. The terms in Equation 9, relate to the accuracy of the inverse metrics defined by $\partial(x, y, z) / \partial(\xi, \eta, \zeta)$. Any inaccuracies contained in the inverse metrics will affect the accuracy of the Jacobian, and the metrics of the grid transformation.

Therefore, for an ideal grid, each of the nine terms in Equation 9 should be zero. A non-dimensional grid quality metric $Q$ is defined, based on these nine terms, and is explained with the aid of Figure 1. In Figure 1, the unfiltered grid point at $(i, j)$ is shifted to the position $\left(i^{\prime}, j\right)$ after applying the filters along the $\xi$ direction. The grid coordinate displacement is defined by,

$$
\hat{\Delta} \xi=\sqrt{\hat{\Delta} x_{i}^{2}+\hat{\Delta} y_{i}^{2}+\hat{\Delta} z_{i}^{2}} .
$$

This quantity is normalised by a local length scale $(\Delta \xi$ in Figure 1$)$ along the curvilinear axis, and is taken as the average grid spacing between neighbouring vertices, and defined as, 


$$
\Delta \xi=\sqrt{x_{\xi}^{2}+y_{\xi}^{2}+z_{\xi}^{2}}
$$

Finally, the grid quality metric for a three-dimensional case can be generalised as,

$$
Q=\sqrt{\left(\frac{\hat{\Delta} \xi}{\Delta \xi}\right)^{2}+\left(\frac{\hat{\Delta} \eta}{\Delta \eta}\right)^{2}+\left(\frac{\hat{\Delta} \zeta}{\Delta \zeta}\right)^{2}}
$$

where larger values of $Q$ indicate larger truncation errors, due to poorly resolved inverse grid metrics and therefore, a low quality grid . The nondimensional variable $Q$ is a field quantity that is defined at all grid points.

\section{Correlation between grid quality metric and solution error}

An important property of a grid quality metric is a strong correlation between the grid quality metric and the solution error for problems where the grid induced error is dominant. To demonstrate and quantify this for the grid quality metric defined in the previous section, a simplified case is used to provide a systematic approach to measure the correlation between

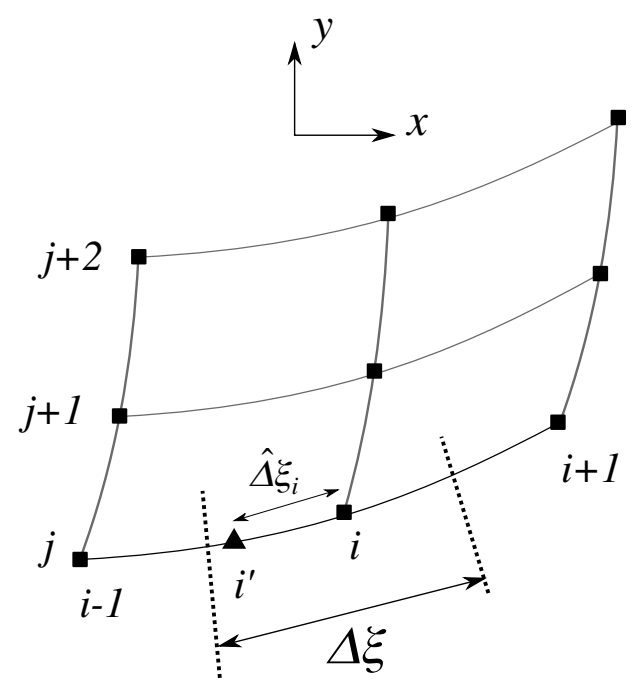

Figure 1: Generalisation of the changes to the grid coordinates induced by spatial filters, on to a two-dimensional curvilinear mesh. Original grid points are highlighted by $\mathbf{\square}$, and the modified coordinate due to the filtering is highlighted by $\mathbf{\Lambda}$. 
the grid quality metric and the solution accuracy. An inviscid vortex core convection problem [24] is used to determine the effectiveness of the grid quality metric $(Q)$ as an indicator of the solution accuracy. The analytical solution to this problem is known and allows the solution errors due to the grid to be calculated.

Initial baseline values of the grid quality metric and solution error are obtained for a uniform grid. Subsequently, different grids with similar grid resolutions are tested to determine the influence of three types of grid features commonly found; abrupt changes in the grid metrics, skewed grids, and grid stretching. Finally, the results from these test cases are used to determine the correlation between the grid quality metric, and the solution accuracy. A strong correlation reflects the effectiveness of the grid quality metric in identifying areas of the grid that are likely to generate large grid-induced errors.

The vortex convection problem is governed by the two-dimensional compressible Euler equations expressed in full conservation form as,

$$
\frac{\partial}{\partial t}\left(\begin{array}{c}
\rho \\
\rho u \\
\rho v \\
\rho e_{T}
\end{array}\right)+\frac{\partial}{\partial x}\left(\begin{array}{c}
\rho u \\
\rho u^{2}+p \\
\rho u v \\
\rho\left(e_{T}+p\right) u
\end{array}\right)+\frac{\partial}{\partial y}\left(\begin{array}{c}
\rho v \\
\rho u v \\
\rho v^{2}+p \\
\rho\left(e_{T}+p\right) v
\end{array}\right)=0,
$$

where $\rho, \vec{u}=(u, v)$, and $p$ are the non-dimensional density, velocity, and the static pressure respectively. The free-stream density and speed of sound, are used to normalise these variables. The dimensions of space are normalised by a unit length. The total energy $e_{t}$ per unit mass, is defined by,

$$
e_{T}=\frac{p}{\rho(\gamma-1)}+\frac{1}{2} u_{i} u_{i}
$$

where $\gamma=c_{p} / c_{v}$ is the ratio of specific heats. An initialised flow consisting of an inviscid vortex core is marched in time by a fourth-order Runge-Kutta scheme [20], using a Courant number of $C F L<0.45$. The flux terms are evaluated by fourth-order accurate spatial schemes [1,23] and the solution is filtered by sixth-order implicit filters [22] at the end of each time-step. The solution is bounded along the edges of the computational domain by a far-field pressure condition, which is evaluated using the Riemann invariants for a one-dimensional flow normal to the boundary. Along multi-block edges with neighbouring block information, central spatial schemes are applied, 
otherwise, biased spatial schemes are applied. The inviscid vortex [23] is defined by,

$$
\begin{aligned}
\rho(x, y, t) & =\rho_{\infty}\left[1-\frac{\gamma-1}{2} \psi^{2}\left(x^{*}, y\right)\right]^{1 /(\gamma-1)}, \\
u(x, y, t) & =u_{\infty}+a_{\infty}(y / R) \psi\left(x^{*}, y\right) \\
v(x, y, t) & =-a_{\infty}\left(x^{*} / R\right) \psi\left(x^{*}, y\right) \\
p(x, y, t) & =p_{\infty}\left(\rho / \rho_{\infty}\right)^{\gamma} \\
\psi(x, y, t) & =\frac{\epsilon \sqrt{\mathrm{e}}}{2 \pi} \exp \left\{-\frac{x^{2}+y^{2}}{2 R^{2}}\right\} \\
x^{*} & =x-x_{0}-u_{\infty} t .
\end{aligned}
$$

The size and strength of the vortex are controlled by the variables $R$ and $\epsilon$, respectively. For the present study $R=1 \mathrm{~m}$ and $\epsilon=0.05$. The propagation speed of the vortex is set by the free-stream Mach number $\left(M_{\infty}\right)$ of 0.5 . As shown in Figure 2(a), the initial position of the vortex is set to $(x, y)_{t=0}=$ $(-3,0)$ and the final solution is obtained at $t=12$. The error field is defined at every grid point by,

$$
E=\frac{\left(\rho-\rho_{\text {exact }}\right)}{\rho_{\text {exact }}},
$$

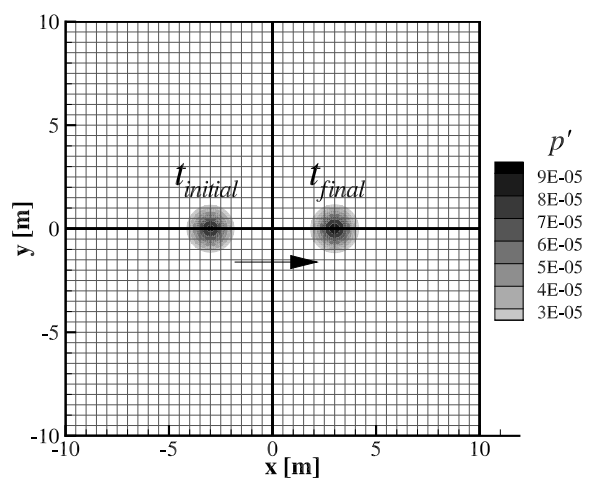

(a)

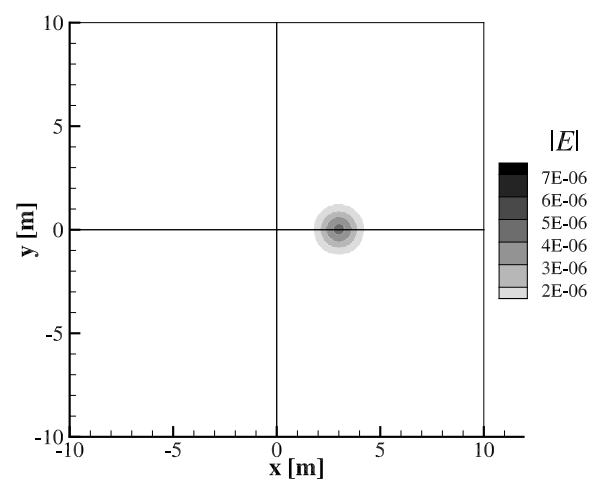

(b)

Figure 2: Four block uniform grid. (a) Pressure disturbance field over-set with every fifth grid line, (b) Density error field at $t=t_{\text {final }}$. 
where $\rho$ and $\rho_{\text {exact }}$ are the numerical and analytical solutions, respectively, of the density field at $t=12$. The grid quality metric is defined by a scalar field $Q$, which is obtained by sixth order explicit [21] and implicit filters [22]. The filters employ central schemes along all block edges where neighbouring block information is available, otherwise, a biased scheme is applied. The integrated values of the solution error and the grid quality are obtained for each case, and are defined by

$$
\begin{aligned}
E_{I} & =\iint_{S}|E| \mathrm{d} x \mathrm{~d} y \\
Q_{I} & =\iint_{S} Q \mathrm{~d} x \mathrm{~d} y
\end{aligned}
$$

where $S$ determines the size of a square integration region. A value of $S=$ $6 \mathrm{~m} \times 6 \mathrm{~m}$ was used for all test cases, except for the uniformly skewed grid. For uniformly skewed cases, a smaller value of $S=3 \mathrm{~m} \times 3 \mathrm{~m}$ was required to ensure that the integration region is confined to the computational domain. For each test the inviscid vortex is convected through the area of poorest grid quality.

\subsection{Uniform mesh}

The uniform mesh illustrated in Figure 2(a) consists of four square domains. Each domain consists of $101 \times 101$ grid points with a uniform grid resolution of $0.1 \mathrm{~m} \times 0.1 \mathrm{~m}$. The half width of the vortex is $R=1 \mathrm{~m}$, and the entire width of the vortex is resolved along 20 grid points. The grid quality metric is uniform, and of the order of $Q(x, y)=O\left(10^{-14}\right)$. The error in the density field, illustrated in Figure 2(b), is of the order of $|E(x, y)|=O\left(10^{-6}\right)$. The integrated values of grid quality and solution error are $Q_{I}=O\left(10^{-9}\right)$, and $E_{I}=O\left(10^{-5}\right)$, respectively. The uniform test case provides baseline measures for the solution error, and verifies that the ideal grid quality metric is extremely small.

\subsection{Abrupt changes in the grid metrics}

Abrupt changes in the grid metrics can be found in complex structured grids, such as a landing gear model [2]. Along multi-block interfaces, a standard central differencing scheme may result in strong grid-induced errors [25]. A generalised Characteristic Boundary Condition (CBC) [25] can overcome 
these numerical issues. In this section, two types of abrupt changes in the grid metrics are tested. The first is an abrupt change in the grid spacing, and the second is an abrupt change in the grid line direction. For these tests the grid quality is measured using central filtering schemes along the block interfaces. However, measures of the numerical accuracy are evaluated from separate solutions obtained using standard central differencing schemes, and by using a CBCs.

\subsubsection{Abrupt change in the grid spacing}

An abrupt change in the grid spacing is imposed by applying the following function to the grid coordinates of the uniform mesh, described in Section 3.1

$$
x^{*}(\xi, \eta)=x(\xi, \eta) A_{x} H(x),
$$

where $(x, y)$ and $\left(x^{*}, y^{*}\right)$ are the original, and modified grid coordinates respectively, and $H(x)$ is the Heaviside function. The constant $A_{x}$ specifies the grid spacing size for all grid points along $x>0$, relative to the original grid spacing of $\Delta x=0.1 \mathrm{~m}$. Figure 3 illustrates the grid quality metric field for two examples for $A_{x}=1.25$ and 2. As shown in Figure 3, the grid quality metric correctly highlights the origin of the grid spacing discontinuity indicated by larger values of the scalar $Q$. As the constant $A_{x}$ is increased, the grid spacing discontinuity is enlarged. Therefore, larger values in the scalar $Q$ are obtained, which signifies a lower grid quality due to truncation errors in the calculation of the grid metrics.

Figure 4 illustrates the numerical errors generated when a standard central differencing scheme is applied across the grid metric discontinuity. These errors act as a non-physical acoustic source. The integrated values of the grid quality $Q_{I}$, and the solution error $E_{I}$ are plotted from seven test cases in Figure 5. In Figure 5, the grid quality measured using explicit and implicit filters are included and show a similar trend with increasing $A_{x}$. The relationship to the solution accuracy is plotted for a grid quality metric calculated by the implicit filters only.

The general trend in Figure 5 indicates that as the grid spacing discontinuity is increased, the grid quality is reduced. This leads to an increase in the truncation errors of the metrics of the grid transformation. When a $\mathrm{CBC}$ is used at the grid metric discontinuity, significantly lower errors are generated. However, a strong correlation between the grid quality metric and 
the numerical error is still obtained. The solution error is also contributed to by the reduction in grid resolution of the vortex in the downstream region with larger $A_{x}$. The abrupt change in the grid spacing with $A_{x}=2$, yields an integrated grid quality of $Q_{I}=O\left(10^{0}\right)$, and generates an integrated error that is two orders of magnitude greater than the baseline uniform case.

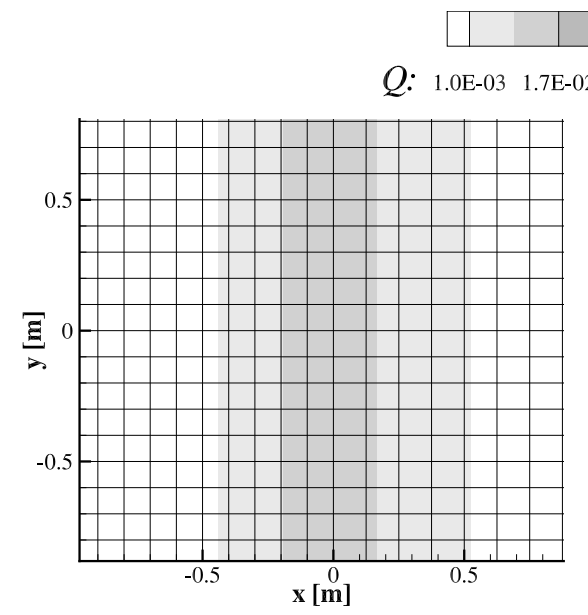

(a)

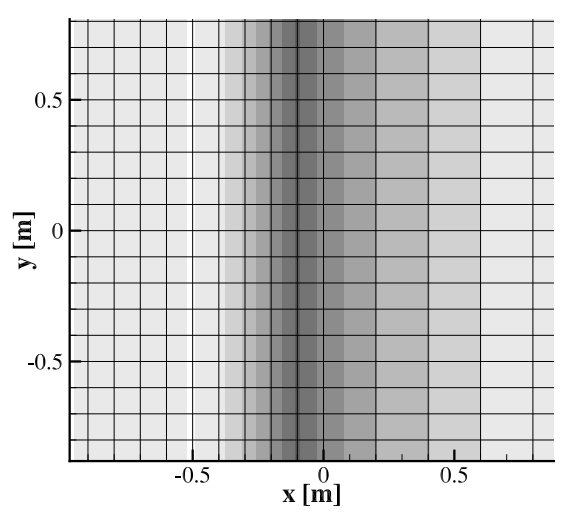

(b)

Figure 3: The effect of an abrupt change in the grid spacing at $x=0$ on the grid quality evaluated by implicit and central filters. (a) $A_{x}=1.25$, (b) $A_{x}=2$. 
$|E|: \quad 5.0 \mathrm{E}-07 \quad 1.4 \mathrm{E}-06 \quad 2.3 \mathrm{E}-06 \quad 3.2 \mathrm{E}-06 \quad 4.1 \mathrm{E}-06 \quad 5.0 \mathrm{E}-06$

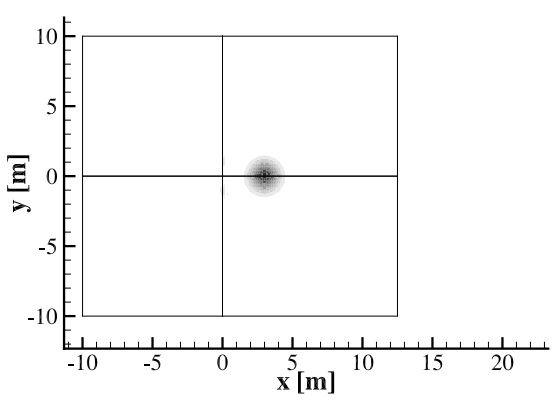

(a)

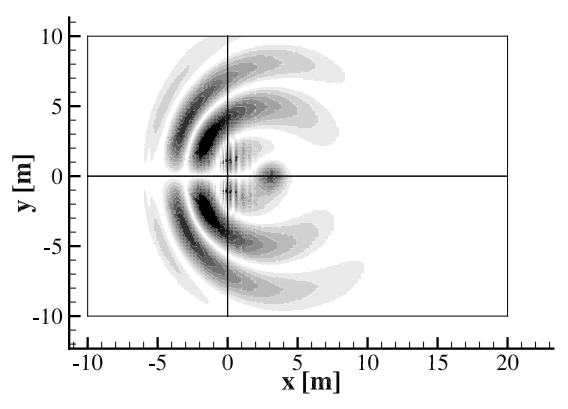

(b)

Figure 4: The effect of an abrupt change in the grid spacing at $x=0$ on the solution error at $t=t_{\text {final }}$, without a characteristic boundary condition. (a) $A_{x}=1.25$, (b) $A_{x}=2$.

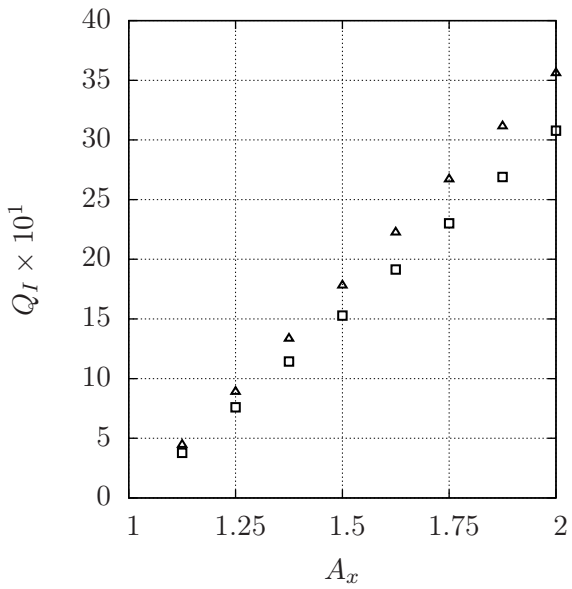

(a)

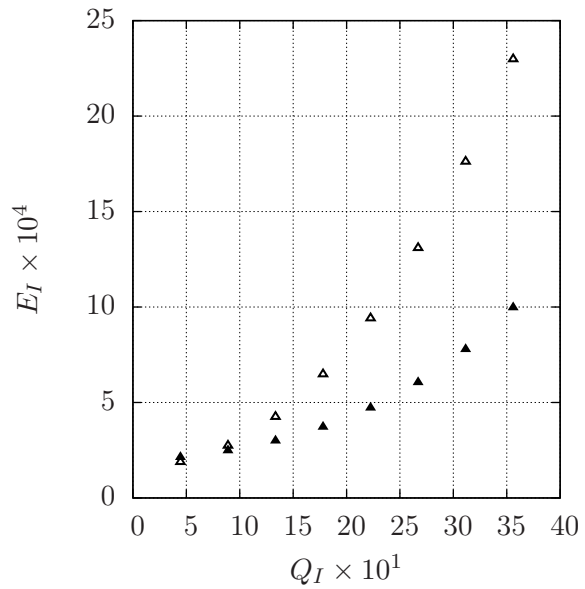

(b)

Figure 5: Effect of an abrupt change in the grid spacing at $x=0$ on the grid quality and on the solution error. (a) The effect of $A_{x}$ on the grid quality measured by explicit $\square$ and implicit $\triangle$ filters, (b) the effect of grid quality on the solution error at $t=t_{\text {final }}$, for a calculation with $\Delta$ and without $\triangle \mathrm{CBC}$. 


\subsubsection{Abrupt change in the grid line direction}

Abrupt changes to the grid line direction are imposed by applying the following function to the grid coordinates of the uniform mesh, described in Section 3.1

$$
y^{*}(\xi, \eta)=y(\xi, \eta)+H(x) A_{y} x(\xi, \eta),
$$

where $(x, y)$ and $\left(x^{*}, y^{*}\right)$ are respectively the original, and modified grid coordinates, and $H(x)$ is the Heaviside function. The constant $A_{y}$ specifies the grid line gradient for $x>0$. Figures 6 and 7 respectively show the effect that changes in the grid line direction may have on the local grid quality, and the solution accuracy, for two examples of $A_{y}$. The trends illustrated in Figure 8 show that as $A_{y}$ increases, larger truncation errors are contained in the inverse metrics of the grid transformation. Therefore, the solution accuracy is reduced. An abrupt change in the grid line direction with $A_{y}=0.875$ that yields an integrated grid quality of $Q_{I}=O\left(10^{\circ}\right)$, generates an integrated error value that is one order of magnitude larger than the baseline uniform grid.

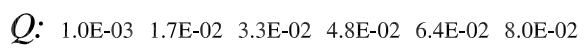

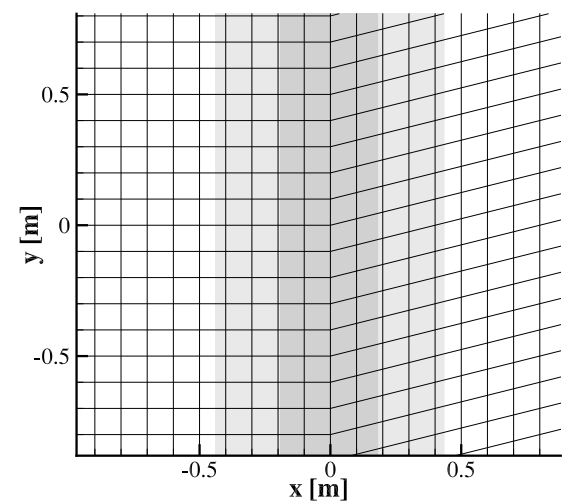

(a)

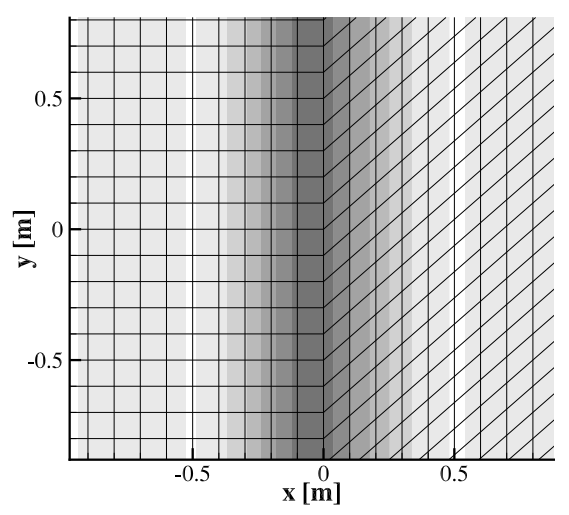

(b)

Figure 6: The effect of an abrupt change in the grid spacing at $x=0$ on the grid quality evaluated by implicit and central filters. (a) $A_{y}=0.25$, (b) $A_{y}=0.875$. 


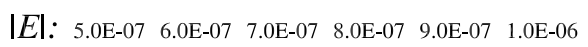

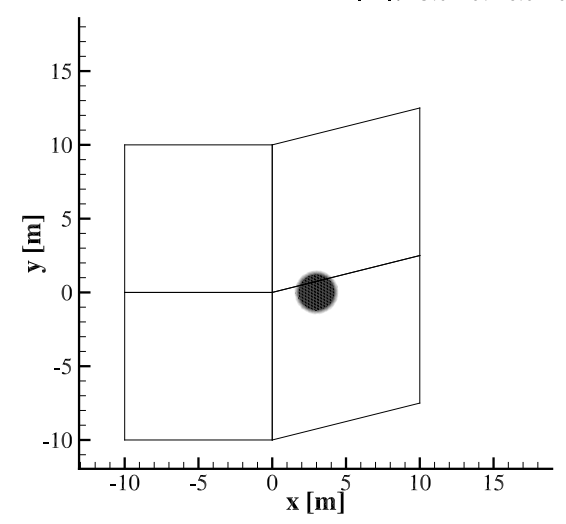

(a)

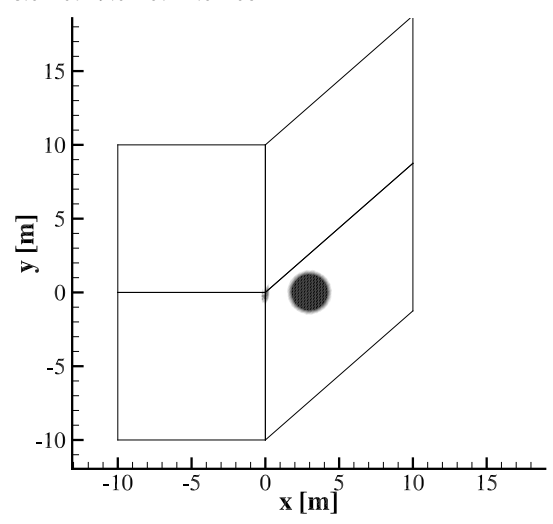

(b)

Figure 7: The effect of an abrupt change in the grid spacing at $x=0$ on the solution error at $t=t_{\text {final }}$. (a) $A_{y}=0.25$, (b) $A_{y}=0.875$.

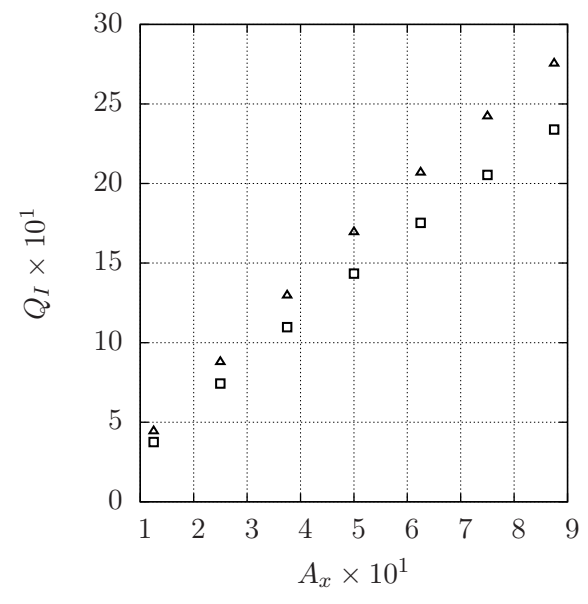

(a)

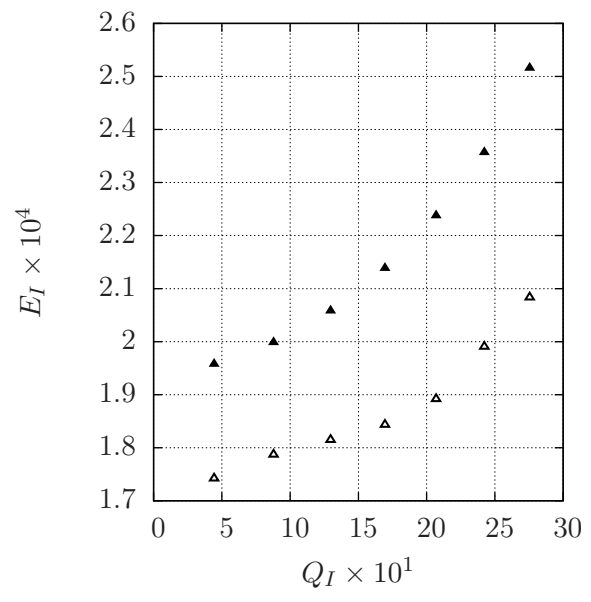

(b)

Figure 8: Effect of abrupt changes in the grid line direction at $x=0$ on the grid quality and the solution error. (a) The effect of $A_{y}$ on the grid quality measured by explicit $\square$ and implicit $\triangle$ filters, (b) the effect of grid quality on the solution error at $t=t_{\text {final }}$, for a calculation with $\triangle$ and without $\triangle \mathrm{CBC}$. 


\subsection{Skewed grid features}

In this section uniform and non-uniformly skewed grids are tested. Uniformly skewed grids have been applied to numerical studies [26, 27], but is a geometric feature that is not often found. Non-uniform grids are much more common for body-fitted structured grids. For this study no discontinuities in the grid metric are present. Therefore, a CBC is not applied in these studies.

\subsubsection{Uniformly skewed grids}

Uniform grid skew is introduced by applying the following function to the grid coordinates of the uniform mesh, described in Section 3.1

$$
x^{*}(\xi, \eta)=x(\xi, \eta)+A_{s} y(\xi, \eta)
$$

where $(x, y)$ and $\left(x^{*}, y^{*}\right)$ are the original, and modified grid coordinates, respectively, and $A_{s}$ is a constant that specifies grid line gradient across the entire domain. The angles between grid lines of constant $\xi$ and $\eta$ are specified by $\arctan \left(A_{s}\right)$. The effect of uniformly skewed grids on the solution accuracy, is shown in Figure 9, for two examples of $A_{s}$. The integrated values of the grid quality and the solution error are illustrated in Figure 10, and are composed from several grids containing varying degrees of the skew angle.

Uniformly skewed grids do not induce any high wave number geometric features. Therefore, it does not affect the dispersion errors in the metrics of the grid transformation. This explains the consistently low levels of the integrated quality metric $Q_{I}=O\left(10^{-11}\right)$. The solution error is unaffected by the skew angle, and the integrated value of numerical error is of the same order of magnitude as the baseline uniform grid.

\subsubsection{Non-uniformly skewed grids}

Non-uniform skew is imposed by applying a trigonometric function to the grid coordinates of the uniform mesh, described in Section 3.1

$$
\begin{aligned}
& x^{*}(\xi, \eta)=x(\xi, \eta)+A_{t} \sin \left(\frac{2 \pi y(\xi, \eta)}{L}\right), \\
& y^{*}(\xi, \eta)=y(\xi, \eta)+A_{t} \sin \left(\frac{2 \pi x(\xi, \eta)}{L}\right)
\end{aligned}
$$




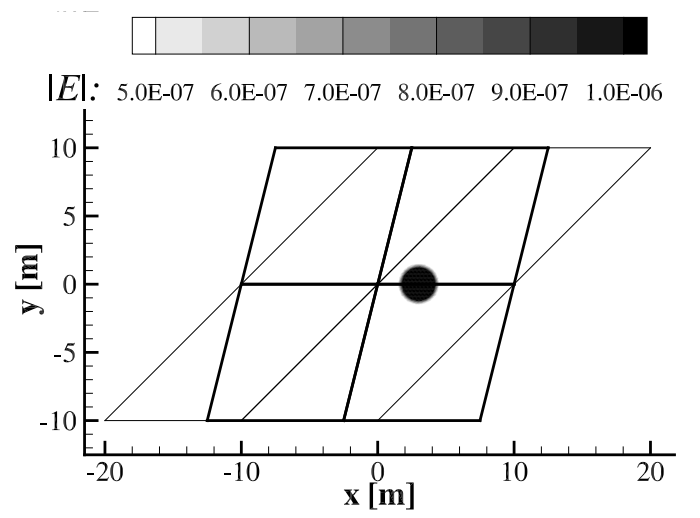

Figure 9: The effect of uniform cell skew in the grid on the solution error at $t=t_{\text {final }}$. Block edges for $A_{s}=0.25$ and $A_{s}=1$ are shown in thick and thin black lines, respectively.

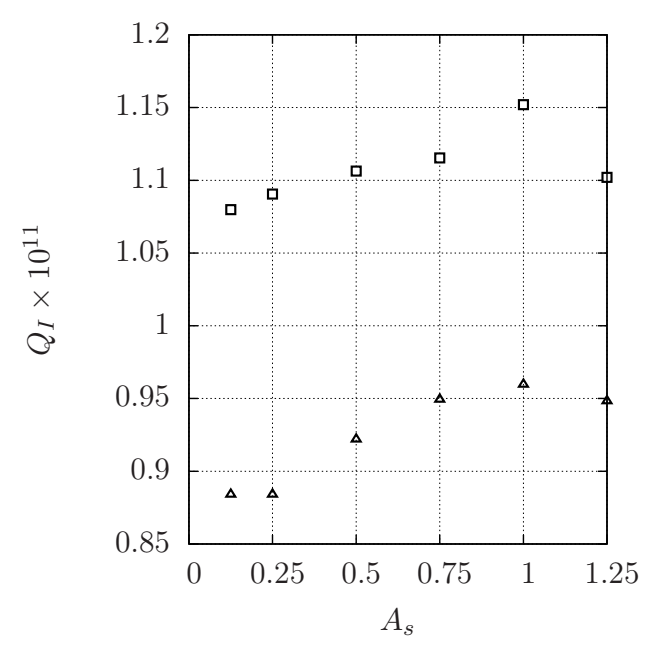

(a)

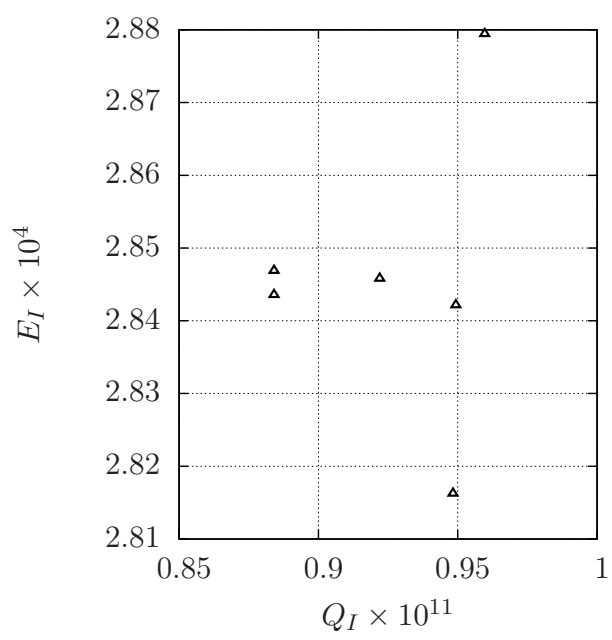

(b)

Figure 10: The effect of uniform skew on the grid quality metric and the solution error, as measured by explicit $\square$ and implicit $\triangle$ filters. (a) The effect of $A_{s}$ on the grid quality, (b) the effect of the grid quality on the solution error at $t=t_{\text {final }}$. 
where $(x, y)$ and $\left(x^{*}, y^{*}\right)$ are respectively the original and modified grid coordinates. The variables $A_{t}$ and $L$ specify the amplitude and wavelength, along which the local skew angle will vary. For the following test cases, the wavelength was set to $L=10 \mathrm{~m}$, and the amplitude $A_{t}$ was varied between different grids.

Figure 11 illustrates the grid quality metric field $Q$, for two examples of $A_{t}$. In these figures the region of lowest grid quality is focused on the areas where the variations in the grid line direction are greatest, instead of the areas where the local skew angle is greatest. The effect of varying $A_{t}$ on the integrated values of the grid quality, and the solution error are illustrated in Figure 12. The integrated grid quality $Q_{I}$ is of the order of $10^{-5}$ and $10^{-8}$ for grid qualities evaluated by the explicit and implicit filters, respectively. Both measure of grid quality indicate that the quality of the tested grids are consistently of high quality. This is due to the high resolution of the sinusoidal grid feature illustrated in Figure 11. The resolution of this grid feature is equivalent to 100 grid points per wavelength. Compared to the baseline uniform case, a grid with $A_{t}=1.5$ generates an integrated solution error that is of the same order of magnitude.

In Figure 12, there is a significant reduction in the correlation, between the grid quality and the solution error, in the region where $Q_{I}<7 \times 10^{-8}$. This indicates the threshold where the grid-induced errors do not dominate. Examples of other sources of error may include the truncation errors in the temporal scheme, or possibly by acoustic reflections due to poor boundary conditions. 


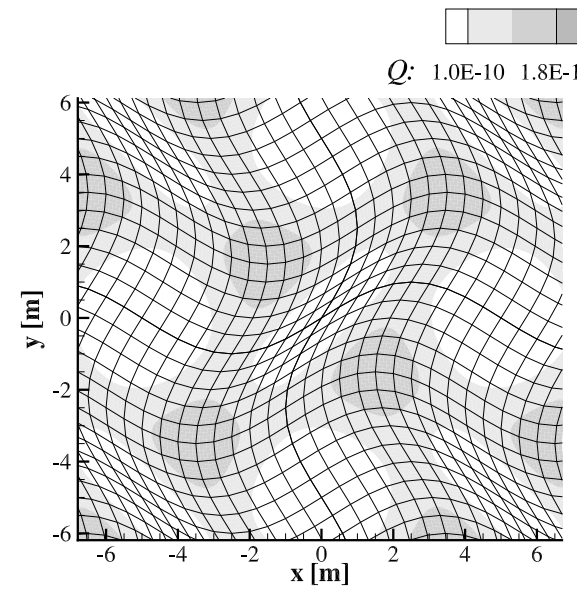

(a)

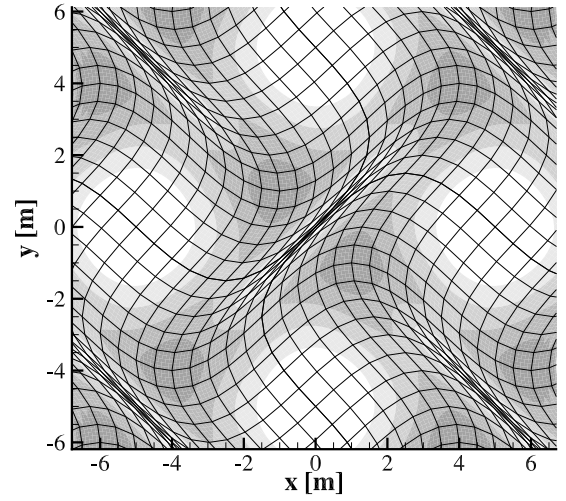

(b)

Figure 11: The effect of non-uniformly skewed grids on the mesh quality evaluated by implicit and central filters overlaid by every fifth grid line. (a) $A_{t}=1$, (b) $A_{t}=1.5$

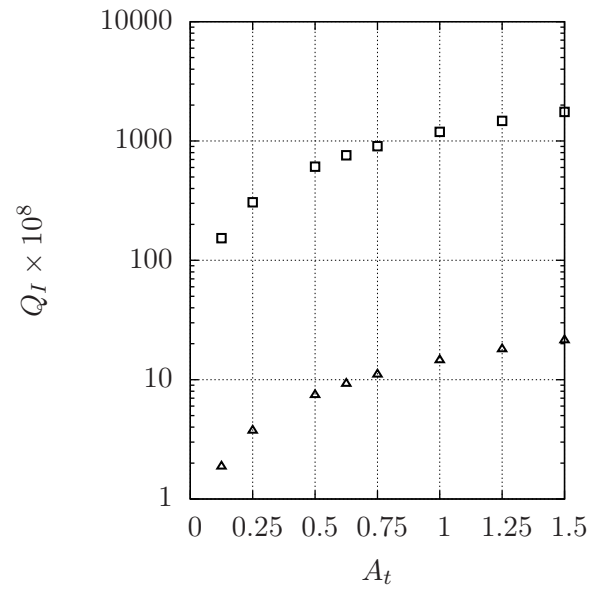

(a)

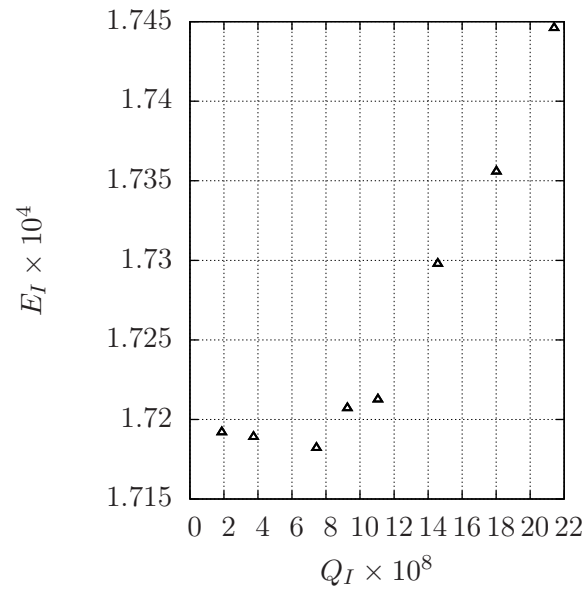

(b)

Figure 12: The effect of non-uniform skew on the grid quality metric and the solution error, as measured by explicit $\square$ and implicit $\Delta$ filters. (a) The effect of $A_{t}$ on the grid quality, (b) the effect of grid quality on the solution error at $t=t_{\text {final }}$. 


\subsection{Grid stretching}

Grid stretching is typically applied to focus the maximum grid resolution to a desired region. Such features are desirable for example in boundary layer regions. Several grid stretching functions have been proposed [14, 15]. A grid with the same uniform transverse spacing $(\Delta y=0.1 \mathrm{~m})$, and distributed stream-wise spacing is defined by the following procedure. In the range where $x \geq 0$ the following grid stretching function, defined in the generalised coordinates, is applied,

$$
\left.\begin{array}{l}
s(\xi)=\frac{\xi}{\xi_{B L}-1} \\
\tilde{x}(\xi)=1-\frac{\tanh (\alpha(1-s(\xi))}{\tanh (\alpha)}
\end{array}\right\} \quad 1 \leq \xi \leq \xi_{B L},
$$

where $\xi_{B L}$ is the number of grid points in the stretched region, $\tilde{x}$ is the normalised grid point distribution function, $\alpha$ affects the level of grid stretching, and $s$ is a uniformly distributed grid of secondary importance. This stretching function is used to define a uniform and stretched region,

$$
\begin{aligned}
& x^{*}(\xi)=A_{h} \tilde{x}(\xi) \\
& \left.x^{*}(\xi)=A_{h}\left[1+\frac{\xi-\max (\xi)}{\xi_{B L}-1}\left(\tilde{x}\left(\xi_{B L}\right)-\tilde{x}\left(\xi_{B L}-1\right)\right)\right]\right\} \\
& 1 \leq \xi \leq \xi_{B L}, \\
& \xi>\xi_{B L},
\end{aligned}
$$

where $A_{h}$ specifies the range $0 \leq x \leq A_{h}$ in which the grid is stretched. Outside this region, the stream-wise grid spacing becomes uniform. For this test case the parameters $A_{h}=1.5 \mathrm{~m}, \xi_{B L}=20$ and $\max (\xi)=101$ are set as constants, and the parameter $\alpha$ is varied between different cases. Since the initialised vortex is located at $x=-3 \mathrm{~m}$, the mesh is mirrored along $x=0$.

In Figure 13, the effect of grid stretching on the solution accuracy is illustrated for two examples. Figure 14 shows that stronger gradients in the trigonometric stretching function will result in a lower integrated grid quality values. Additionally, grids with lower grid quality tend to generate larger numerical errors. Compared to the baseline uniform case, a grid with

$\alpha=3.5$ generates an integrated solution error that is one order of magnitude larger. 
$|E|: \quad 5.0 \mathrm{E}-07 \quad 1.4 \mathrm{E}-06 \quad 2.3 \mathrm{E}-06 \quad 3.2 \mathrm{E}-06 \quad 4.1 \mathrm{E}-06 \quad 5.0 \mathrm{E}-06$

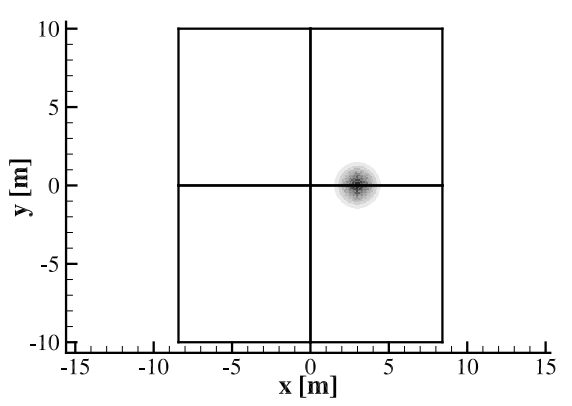

(a)

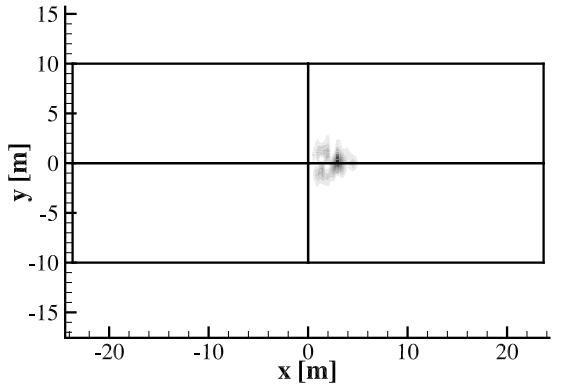

(b)

Figure 13: The effect of an abrupt change in the grid spacing at $x=0$ on the solution error at $t=t_{\text {final }}$, using central differencing schemes. (a) $\alpha=0.5$, (b) $\alpha=3.5$.

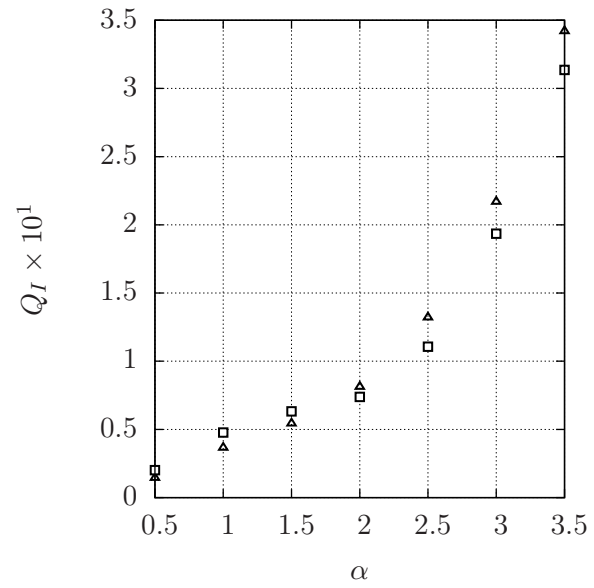

(a)

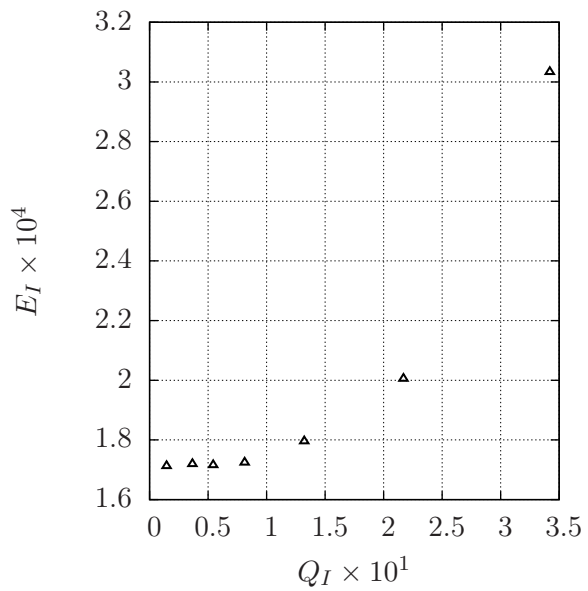

(b)

Figure 14: The effect of non-uniform skew on the grid quality metric and the solution error, as measured by explicit $\square$ and implicit $\Delta$ filters. (a) The effect of $A_{t}$ on the grid quality, (b) the effect of grid quality on the solution error at $t=t_{\text {final }}$. 


\subsection{The effectiveness of the grid quality metric}

From the test case results, where grid errors dominate, it was shown that the grid quality metric is a monotonic function of the solution error. The correlation coefficient $\left(C_{Q E}\right)$, between the integral measures of the grid quality metric $\left(Q_{I}\right)$ and the solution error $\left(E_{I}\right)$, is calculated as follows [28],

$$
C_{Q E}=\frac{\left\langle Q_{I} E_{I}\right\rangle}{\left[\left\langle Q_{I}^{2}\right\rangle\left\langle E_{I}^{2}\right\rangle\right]^{1 / 2}},
$$

where \langle\rangle denotes the variance. In Table 1 the correlation coefficient is listed, for the test cases containing particular grid features. This is a measure of the ability of the grid quality scalar $Q$ to identify areas of the grid that are likely to generate significant grid-induced errors. In Table 1 the correlation is listed for four cases. The first and third columns corresponds to grid qualities measured by explicit and implicit sixth-order filters. The grid quality measures are obtained without biased boundary schemes along the grid metric discontinuities. The second and fourth column measure the correlation between the two types of filters tested and a numerical solution obtained with the use of a Characteristic Boundary Condition (CBC) along grid metric discontinuities. Geometric grid features commonly found in structured grids are; grid metric discontinuities, skewness, and stretching.

Table 1: Correlation coefficient between the grid quality metric and the solution error.

\begin{tabular}{ccccc}
\hline Grid features & $\begin{array}{c}\text { Explicit } \\
\text { filter }\end{array}$ & $\begin{array}{c}\text { Explicit filter } \\
\mathrm{w} / \mathrm{CBC}\end{array}$ & $\begin{array}{c}\text { Implicit } \\
\text { filter }\end{array}$ & $\begin{array}{c}\text { Implicit filter } \\
\mathrm{w} / \mathrm{CBC}\end{array}$ \\
\hline Grid spacing discontinuity & 0.971 & 0.963 & 0.970 & 0.962 \\
Grid direction discontinuity & 0.955 & 0.969 & 0.954 & 0.968 \\
Uniform skewness & 0.472 & $\mathrm{~N} / \mathrm{A}$ & 0.128 & $\mathrm{~N} / \mathrm{A}$ \\
Non-uniform skewness & 0.921 & $\mathrm{~N} / \mathrm{A}$ & 0.921 & $\mathrm{~N} / \mathrm{A}$ \\
Grid stretching & 0.932 & $\mathrm{~N} / \mathrm{A}$ & 0.913 & $\mathrm{~N} / \mathrm{A}$ \\
\hline
\end{tabular}

From Table 1 the following observations are found. The grid-induced grid metric truncation errors by uniformly skewed grids is insignificant, and also uncommon in applications. Grid stretching, non-uniformly skewness, and grid metric discontinuities are more common in practice, and consistently show a high correlation coefficient. This shows that the proposed grid quality metric is effective in identifying a priori regions of the grid that may generate large grid-induced errors. 
The most significant grid-induced errors are generated by an abrupt change in the grid spacing. This can be a result of an incorrect parameter definition in the grid point distribution function applied along a block edge, and caused by human error. Using the proposed grid quality metric, these regions can be identified easily. If these regions cannot be easily resolved, they may be treated by a characteristic boundary condition.

Another significant source of grid-induced errors is excessive grid stretching in Table 1. The grid quality metric highlights the regions of the grid that are most likely to be the source of significant grid metric errors in the solution. The grid quality metric also correlates strongly to the solution error for these cases. In these test cases, the parameters that control the amplitude of the grid stretching are spread across a range from very low to very large, values. As a result, the solution error asymptotes as the extent of grid stretching tends towards a uniformly spaced grid. At this limit, the sources of grid metric errors become less significant, compared to other sources of numerical errors.

An effective grid quality metric should correlate to the solution accuracy, and additionally be invariant to the grid scale and the grid orientation. These additional desirable properties are demonstrated in Figure 15. Baseline levels of the desirable grid quality can be established for different solvers by a series of test cases. These levels can be applied to any grid, regardless of its scale or orientation. The threshold between a grid of satisfactory and unsatisfactory quality will vary between solvers, and depend primarily on the resolution characteristics of the spatial schemes employed. Factors such as the order of the scheme, and the amount of artificial dissipation, will additionally affect this threshold.

\section{Conclusion}

Body-fitted, curvilinear grids that are manually generated may contain regions of large grid-induced errors. These regions are typically removed by an iterative process where solution fields are analysed, followed by some grid modifications. This procedure may be lengthy until a satisfactory grid is obtained. Larger grid-induced errors may be suppressed by the use stronger spatial filters, or artificial dissipation. However, stronger filters will damp fine scale features in the solution field, and therefore degrade the fidelity of the solution. A high quality grid may be generated much more efficiently if an effective grid quality metric is defined to aid the user in creating a grid. 


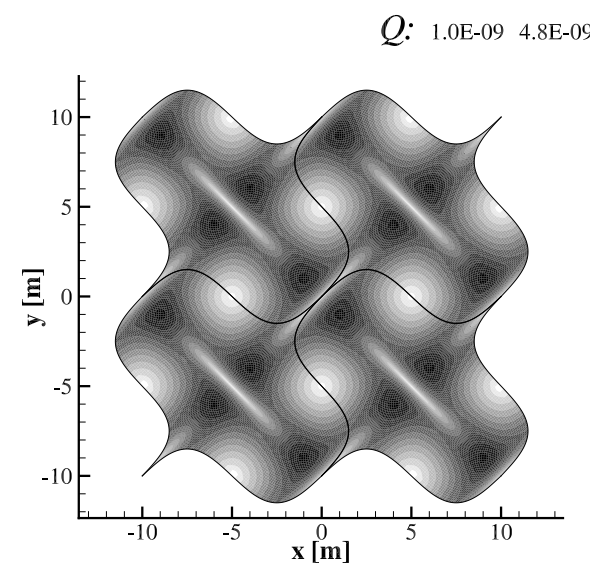

(a)

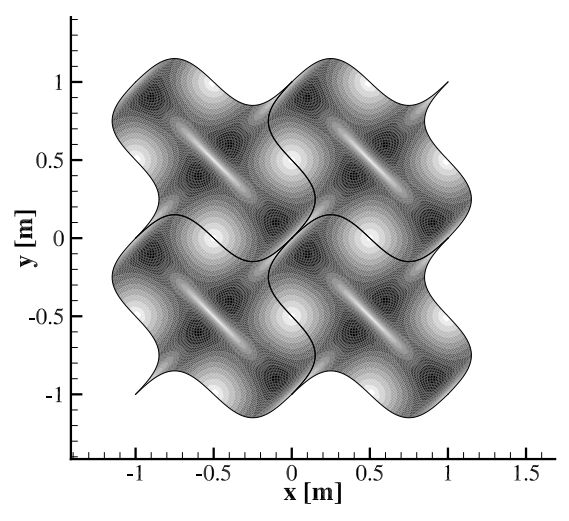

(b)

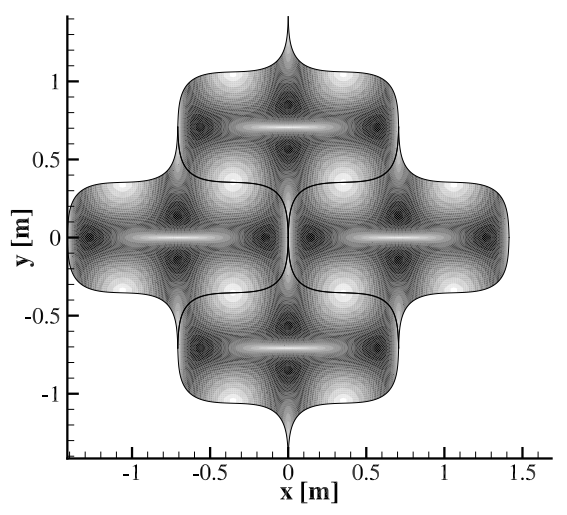

(c)

Figure 15: The effect of grid scale and grid orientation on the grid quality metric $Q$ computed by explicit filters for a uniform grid morphed by Equation 22 with $A=1.5$. (a) Original grid (b) original grid scaled by 0.1 , (b) original grid scaled by 0.1 and rotated by 45 degrees. 
For time-invariant structured grids employing finite differencing schemes, the grid-induced errors are due to two sources. The first is related to the grid resolution of the solution field, and resolved by a grid convergence study. The second source of error is independent of the solution field and is affected by the accuracy of the grid transformation metrics.

The accuracy of the grid transformation metrics is affected by the inverse metrics, which are measures of the spatial derivatives on the grid along the generalised coordinates. The truncation errors contained in the inverse metrics are generated by the spatial schemes. Fourier analysis shows that the dispersion errors, by spatial schemes, have similarities to the transfer function of spatial filters. This similarity is exploited to define a grid quality metric that can be used to identify areas in the mesh that are likely to generate significant grid-induced errors. These areas can be identified clearly and remedied.

Several test cases on different grids show that the proposed grid quality metric is strongly correlated to the solution accuracy, under the condition that the grid-induced errors are the most significant sources of error. Therefore, improvements to the grid quality metric are very likely to result in an improved solution accuracy. This method improves the fidelity of high-order solvers by minimising the application of additional artificial dissipation that is required to ensure numerical stability, and reduces the amount of computational resources, and time, required for the iterative grid generation procedure to obtaining a satisfactory grid.

The grid quality metric proposed is also normalised, which makes the scalar field independent of grid scale or grid orientation. Therefore guidelines on the desired levels of the scalar can be evaluated and applied to any grid. These guidelines will vary between solvers due to the differences in the resolution characteristics of the numerical methods employed. If low-order filtering schemes are used to assess the grid quality, areas of the grid that may not necessarily induce significant grid-induced errors, will be additionally highlighted. Therefore, the transfer function of the filters, should closely match the dispersion relation of the spatial differencing scheme in the solver. With these guidelines defined, areas of the grid likely to generate significant truncation errors in the metrics of the grid transformation can be identified and resolved, all without any knowledge of the solution field. 


\section{Acknowledgements}

The authors would like to thank Meng Wang and James Gill for their comments and advice. 


\section{Bibliography}

[1] J. W. Kim, R. D. Sandberg, Efficient parallel computing with a compact finite difference scheme, Computers \& Fluids 58 (15 April 2012) (2012) 70-87. doi:10.1016/j.compfluid.2012.01.004.

[2] W. Liu, J. W. Kim, X. Zhang, D. Angland, B. Caruelle, Landinggear noise prediction using high-order finite difference schemes, Journal of Sound and Vibration 332 (14) (2013) 3517-3534. doi:10.1016/j.jsv.2013.01.035.

[3] C. W. Mastin, Error induced by coordinate systems, Applied Mathematics and Computation 10-11 (1982) 31-40. doi:10.1016/00963003(82)90186-2.

[4] J. W. Kim, D. J. Lee, Characteristic interface conditions for multiblock high-order computation on singular structured grid, AIAA Journal 41 (12) (2003) 2341-2348.

[5] M. R. Visbal, D. V. Gaitonde, On the use of higher-order finite-difference schemes on curvilinear and deforming meshes, Journal of Computational Physics 181 (1) (2002) 155-185. doi:10.1006/jcph.2002.7117.

[6] S. J. Alter, A structured-grid quality measure for simulated hypersonic flows, in: 42nd AIAA Aerospace Sciences Meeting and Exhibit, 5-8 January, Reno, Nevada, no. AIAA 2004-0612, 2004.

[7] J. F. Dannenhoffer, Correlation of grid quality metrics and solution accuracy for supersonic flows, in: 50th AIAA Aerospace Sciences Meeting, Nashville, Tennessee, no. AIAA 2012-0610, 2012.

[8] T. Colonius, S. K. Lele, Computational aeroacoustics: progress on nonlinear problems of sound generation, Progress in Aerospace Sciences 40 (6) (2004) 345-416. doi:10.1016/j.paerosci.2004.09.001.

[9] P. M. Knupp, Elliptic grid generation, Society for Industrial and Applied Mathematics 17 (6) (1996) 1475-1490. doi:10.2514/3.14240.

[10] J. F. Middlecoff, P. D. Thomas, Direct control of the grid point distribution in meshes generated by elliptic equations, AIAA Journal 18 (6) (1979) 652-656. doi:10.2514/3.50801.

URL http://doi.aiaa.org/10.2514/3.50801 
[11] D. Lee, M. Tsuei, A formula for estimation of truncation errors of convection terms in a curvilinear coordinate system, Journal of Computational Physics 98 (1) (1992) 90-100.

[12] S. Sankaranarayanan, M. L. Spaulding, A study of the effects of grid nonorthogonality on the solution of shallow water equations in boundaryfitted coordinate systems, Journal of Computational Physics 184 (1) (2003) 299-320. doi:10.1016/S0021-9991(02)00042-6.

[13] P. M. Knupp, Remarks on mesh quality, in: 46th AIAA Aerospace Sciences Meeting, no. AIAA 2008-933, 2008.

[14] M. Vinokur, On one-dimensional stretching functions for finite-difference calculations, Journal of Computational Physics 50 (2) (1983) 215-234. doi:10.1016/0021-9991(83)90065-7.

[15] J. F. Thompson, Z. U. A. Warsi, C. W. Mastin, Chapter 5, in: Numerical grid generation: foundations and applications, North-Holland, New York, 1982, Ch. 5, p. 483.

[16] X. Deng, M. Mao, G. Tu, H. Liu, H. Zhang, Geometric conservation law and applications to high-order finite difference schemes with stationary grids, Journal of Computational Physics 230 (4) (2011) 1100-1115. doi:10.1016/j.jcp.2010.10.028.

[17] S. K. Lele, Compact finite difference schemes with spectral-like resolution, Journal of Computational Physics 103 (1) (1992) 16-42. doi:10.1016/0021-9991(92)90324-R.

[18] R. Vichnevetsky, J. Bowles, Fourier analysis of numerical approximations of hyperbolic equations, Society for Industrial and Applied Mathematics 26 (3) (1984) xii +135.

[19] G. Ashcroft, X. Zhang, Optimized prefactored compact schemes, Journal of Computational Physics 190 (2) (2003) 459-477. doi:10.1016/S00219991(03)00293-6.

[20] F. Hu, M. Hussaini, J. Manthey, Low-dissipation and low-dispersion RungeKutta schemes for computational acoustics, Journal of Computational Physics 124 (1) (1996) 177-191. 
[21] O. V. Vasilyev, T. S. Lund, P. Moin, A general class of commutative filters for LES in complex geometries, Journal of Computational Physics 146 (1) (1998) 82-104. doi:10.1006/jcph.1998.6060.

[22] J. W. Kim, High-order compact filters with variable cut-off wavenumber and stable boundary treatment, Computers \& Fluids 39 (7) (2010) 1168-1182. doi:10.1016/j.compfluid.2010.02.007.

[23] J. W. Kim, Optimised boundary compact finite difference schemes for computational aeroacoustics, Journal of Computational Physics 225 (1) (2007) 995-1019. doi:10.1016/j.jcp.2007.01.008.

[24] H. C. Yee, N. D. Sandham, M. J. Djomehri, Low-dissipative high-order shock-capturing methods using characteristic-based filters, Journal of Computational Physics 150 (1) (1999) 199-238.

[25] J. W. Kim, D. J. Lee, Generalized characteristic boundary conditions for computational aeroacoustics, AIAA Journal 38 (11) (2000) 2040-2049.

[26] E. Peers, X. Zhang, J. W. Kim, Patched characteristic interface condition for high-order multiblock aeroacoustic computation, AIAA Journal 48 (11) (2010) 2512-2522. doi:10.2514/1.J050130.

[27] T. Gerz, U. Schumann, S. E. Elghobashi, Direct numerical simulation of stratified homogeneous turbulent shear flows, J. Fluid Mech. 200 (1989) 563-594.

[28] S. B. Pope, Turbulent Flows, in: Book, Vol. 1, Cambridge University Press, Cambridge, 2000. doi:10.1088/1468-5248/1/1/702. 
